\title{
Inner Light Perception as a Quantum Phenomenon-Addressing the Questions of Physical and Critical Realisms, Information and Reduction
}

\begin{abstract}
Ravi Prakash* and Michele Caponigro ${ }^{\dagger}$
Abstract

Subjectivity or the problem of 'qualia' tends to make the accessibility and comprehension of psychological events intangible especially for scientific exploration. The issue becomes even more complicated but interesting when one turns towards mystical experiences. Such experiences are different from other psychological phenomena in the sense that they don't occur to every one, so are difficult to comprehend even for their qualifications of existence. We conducted a qualitative study on one such experience of inner-light perception. This is a common experience reported by meditators of all kinds. However, we chose to study this phenomenon in Vihangam Yoga practitioners because of frequent occurrence of this experience in them as well as their reports of having it for hours at a stretch. During this study, it was noted that it arose many questions there we need to answer not only to explain such phenomena but also for having a better understanding of philosophy of science. In the search for these answers, we proceeded towards another complicated branch of science, quantum mechanics. Our present work is about creating an interface between a unique subjective phenomenon and principles of philosophy as well as of quantum mechanics. We explore the constructs of physical and critical realisms and their coincidence, quantum information theory and the measurement problem of Copenhagen interpretation and their possible applications in such an experience. In this endeavour, we also address the possibility that inner-light perception as experienced by Vihangam Yogis is a quantum event in brain. For this purpose, we specifically analyse the Zeilingers information concept and try to apply it to this phenomena.
\end{abstract}

Key Words: inner light perception, critical realism, physical realism, quantum reduction, Copenhagen interpretation

NeuroQuantology 2009; 1: 188-197

\section{Introduction}

Psychological phenomena have always been surrounded by the aura of subjectivity. Popularly known by the problem of "qualia", subjectivity definitely makes the accessibility

Corresponding author: Ravi Prakash

Address: *Central Institute of Psychiatry, India and ${ }^{\dagger}$ University

of Bergamo - via Salvecchio 19 - 24129 Bergamo - Italy

e-mail: drravi2121@gmail.com

ISSN 13035150 of data of psychological events more difficult and intangible (Dennet, 1988; 1991). The problem deepens if we turn towards exploration of mystical experiences. It is difficult to consider mystical experiences as real because of our inability to experience them. But does this inability on our part disqualify them for being real? We recently conducted a qualitative study on the inner-light perception condition of Vihangam Yoga 
practitioners. During the study, several questions arose that posed difficulty at the philosophical level of science. These were the questions related to concepts of realism and information that are central to science as a whole. Interestingly, we found parallels of this phenomena and questions similar to these in the drastically-evolving field of quantum mechanics. Our present work is an endeavour to create an interface between subjective experiences specifically ones like inner-light perception and the principles of quantum mechanics both applicable to science as a whole. We start with a brief description of our qualitative study on this phenomena. Then we move on to address the questions raised by this condition. We will see that these questions are so central to the philosophies of science that for answering them, we need to acknowledge the concepts of both critical and physical realisms as well as that of information. As we try to answer these questions, it will be observed that inner-light perception can be theorised as a quantum phenomena in brain. For this, we will specifically focus on the Zeilingers ideas of information. Finally, we will make a passing reference on the issue of measurement problems in quantum mechanics as proposed in the Copenhagen interpretation and the similarity of this problem in the measurement of inner-light perception condition.

\section{Interpretative Phenomenological Analysis}

Interpretative phenomenological analysis is a relatively new method of qualitative analysis which has its roots in critical realism (Bhaskar, 1978) and social cognition paradigm (Fiske, 1991). Its central concern is to explore how people make sense of their experiences. It utilises the empirical level of critical realism so that it assumes that there are real structures which exist independently of our experience - but we can only access the circular relationship between reality and discourse. The participant's 'lived experience' is coupled with a subjective and reflective process of interpretation, in which the analyst explicitly enters into the research process. Interpretative phenomenological analysis has been widely used for the purpose of qualitative analysis of several phenomena including genetics, sexual disorders, dementia etc (Reid, 2005).

\section{Interpretative Phenomenological Analysis of Inner-Light Perception of Meditation}

In contrast to the objective aspects, subjective states of meditation have been much less explored. One of the reasons for this less indulgence seems to be the implausible and mystic nature of these subjective experiences. Out of body experiences, multimodal sensory experiences, visualisation of sceneries of totally new places or persons, are examples of such experiences. However, in addition to such difficult-to-describe experiences, less complicated experiences like "light perception" have also been described widely in the literature. Several meditators give accounts of perceiving some luminance while doing meditation. Often these kinds of experienced are given spiritual connotations. In spite of such widespread description, it has not been explored scientifically. We conducted a study which was aimed at exploring these subjective experiences using qualitative methodology. We explored the subjective mental states of the focus group constituted by Yogis practicing the meditative procedure of Vihangam Yoga, who stated explicitly of perceiving some sort of luminance while doing the meditative procedure with eyes closed. Vihangam Yoga is a very ancient Indian meditation procedure, whose mentions have been reported in the most ancient Indian sacred texts of Vedas(Prasad, 1989; Prasad, 1994). There were two reasons for which this meditative procedure was chosen for present study: (a) The authors had visited get together meetings of 4 meditation sects (satsanga) and had explored for the number of meditators who claimed of seeing light in their meditative practices. Of the four groups, the number of meditators reporting of luminance perception ( $n=41$ out of 128 ) far exceeded those of other groups $(n=13$ of $69, n=7$ of 58 and $\mathrm{n}=22$ of 150 meditators attending their individual meetings). Further among them, accounts of light perception for hours at a stretch were only reported from the groups of Vihangam Yogis. (b) The meditative procedure is unique in the sense that it is not 
predominantly concentrated in one part of the country, rather has been practiced all across the country. For this qualitative analysis, we used the technique of interpretative phenomenological analysis. In this study, the following themes were obtained (Prakash et al., article in press):

[1] Uniqueness of the nature of light

[2] Emotional and altered identity experiences during light perception

[3] Explanations of the source of light

[4] Change in outlook towards world and associated changes in thinking

\section{Questions Raised From The Inner-Light Perception Condition}

While studying the inner-light perception experiences by Yogis, we came face to face with many problems which are actually questions regarding methodology of science and the concepts of reality. These questions became our impetus for us to present these questions for discussion. Here we address a few of them:

1) Is the inner-light perception of meditation a scientific phenomena? Actually there are two answers to this question. One is what science is perceived by the common man. I think he will like to discard such an experience out right because it does not have any definition and because it is very difficult to comprehend how an individual can see light by eyes closed. So he will tend to think that it not only does not have a definition of its own but it also violates other laws of basic optics. So it is fair enough to call it non-scientific. If we go by the Karl Poppers falsifiability criteria, we will probably end up saying that such a state cannot be tested in a way by which it can be falsified, so it is not scientific. So we end up concluding that it is not scientific and thus we cannot explore it further by scientific means/ methodologies.

2) Is meditation a scientific phenomena? The answer to the earlier question may seem very simple but it has facets which don't allow it to remain that simple. The meditators claim that every one who does meditation will have this experience of "inner-light perception" once he reaches a level of expertise in the same. His Holiness Sadguru Sadafaldev Ji Maharaj, founder of this Vihangam Yoga Organisation, gave an explicit version of the ability of this meditation technique to make the meditators reach the highest level of inner-light if the meditators would practice it regularly: "Yadi Vidhiwat tum sadhan Kariho Amar lok pohunghaunga" (Meaning: If thou do the meditation as has been taught by me, you will reach the divine light of god). Thus it gives us a method to falsify itself. Now if we go by Karl Poppers philosophy, these experiences should be called scientific because now we have a method of testing it in such a way that can falsify it.

3) Is the inner-light perception condition empirical level of realism? The fact that we utilised a methodology based on the principles of critical realism for studying this phenomena evidences the fact that we moved towards a different philosophy of science for studying it. Now we come to a very pertinent question of this topic. Interpretative phenomenological analysis was never used to study this condition. Could this have been because it is just too mystical or because it really does not comply with the laws of critical realism. The empirical level of critical realism has been quoted as the level of realism in which the subject experiences the perception. So here, it can be deduced that the inner-light perception is the empirical level of realism whereas the process of meditation is the actual level of realism, and the realistic level would still be unknown. Is it really so? We really do not know and the reason for bringing this topic to fore was to ignite an argument over it.

4) What next? In spite of these unanswered questions we try to move to the next level of scientific analysis of this phenomena. Suppose we try to find out the mechanisms of this inner-light perception phenomena, is it possible to do so? Perhaps this question is too easy to answer. One could easily say 
no because it cannot be proved to exist. So we turn towards a more complicated question. How can we prove that this state does not exist? Is there a method for it? What current concepts of science and realism have to say for it?

\section{The Entry of Critical Realism}

Among the major scientific revolutions of the past century, origin of the philosophy of critical realism holds a special position. The theory of critical realism has in general acquired a global acceptance and its adaptations are increasing to cover different aspects of realism in different branches of science including qualitative studies like Interpretative phenomenological analysis and cosmology (Bhaskar, 1978). The scientific theories which are grounded on critical realism aim primarily on the structures and mechanisms of the world rather than observable empirical events, as it is believed that the relationship between them is only contingent (Bhaskar, 2008). The differentiating feature of critical realism philosophy from empiricist and positivistic philosophies of science is that critical realism openly accepts the fact that these structures and mechanisms may not be accessible to sense experience (Tsang, 1999). A short explanation of the theory is as follows:

a) The philosophy propounds that there are three levels of reality: These are the empirical, the actual and the real levels. The empirical level of reality is what the subjects experience by perceiving any event. The actual level is the one constituted by events which are logically prior to the experiences. These events are caused by the powers of things- powers that exist even when they are not causing events. The real is constituted by those mechanisms that generate the series of events that constitute the actual level of reality. Thus the real is constituted by those mechanisms that generate the series of events that constitute the actual whereas the empirical, in turn, consists of experiences of certain events (Reed, 2005). Reaching these higher levels of reality has been called as Transcendental realism in the sense that for reaching the real level, one has to transdent the empirical and actual levels.

b) The second important aspect of critical realism is what has been called as critical naturalism. This theory underscores the fact that before we can know how we know, we need to have an idea how we interact with that world in such a way as to acquire knowledge of it (Collier, 1994). The ontological orientation of this theory leads us towards an inquiry into the properties that societies possess while the epistemological orientation leads us to an engagement with how these properties make them possible objects of knowledge for us. This theory emphasises the fact that for acquisition of idea of what people and society actually are, it is necessary to focus on the implicit knowledge we possess by virtue of being people and thus social beings.

\section{The Entry of Physical Realism}

Unfortunately, the series of questions don't seem to end here. We will try to address two harder questions, which are perhaps harder than the hard problem of inverse correlation in consciousness studies. Is inner light perception only a subjective state or is there an element of physical realism to it? Perhaps it may sound too absurd and self-contradictory in first instance. But a closer look may make it clearer. We actually try to find out whether the innerlight perception complies with current concepts of physical realism? Could it be that the subjective experience is some quantum state of physical realism? Do we need to involve the concepts of quantum epistemology to analyse this phenomena? We will try to find answers to these questions by exploring the concepts of physical realism. Out of the several types of physical realism stated in the literature, we will 
focus on the ontological physical realism which deals with the questions of existence and possibilities of mind independent worlds (Vernette, 2006). There have been again many lines of thoughts about the ontological physical realism, of which we consider Einstein's, Rovelli's, D'Espagnant's and Zeilinger's concepts. We will try to fit the condition of inner light perception in each of these concepts and will try to investigate whether it is possible to call this state physically real.

\section{Inner Light Perception In EInstein's Position}

Einstein gave the following proposition for the fulfilment of criteria of physical realism:

"If, without in any way disturbing a system, we can predict with certainty (i.e., with probability equal to unity) the value of a physical quantity, then there exists an element of physical reality corresponding to this physical quantity."As mentioned by Vernette and Caponigro (2006), here exists a condition of forced isomorphism between physical reality and the physical quantity.

Now implying this theoretical conjuncture to our inner-light perception condition, it is difficult to assign an element of physical reality to the condition. We definitely cannot equate the subjective experience of inner-light perception with some physical quantity (physical quantity is in essence an object with objective properties which are independent of the measurements). However, we can have quantitative evidence of this state by neuroimaging measures. But as per this theory, there then will not be the physical realism in the subjective experience but in the states of brain because only the states of brain can be equated with physical quantity.

Again even if we consider the subjective inner-light-perception as a physical quantity, we can not predict the value of it with certainty which again discards its possibility of having element of physical realism. So this theory discards the possibility of subjective experiences like this one of being physically real.

\section{Inner Light Perception In Rovelli's Position}

Rovelli's position can be seen as a drastic departure from Einstein's position. He propounded the following theory:

"The physical reality is taken to be formed by the individual quantum events through which interacting systems (objects)affect one another. Quantum events exist only in interactions and the reality of each quantum event is only relative to the system involved in the interaction". (Rovelli, 1996)

This system rejects the importance of assignment of any system as observer or as observed. All the systems are seen in relation to each other. All the systems can provide observers and physics is concerned about the information that any system can have. The exchange of information is possible between the systems but this exchange is again a quantum information process.

This theory is enlightening in that it provides a possibility that the perception of inner light can be seen as a information exchange between two systems of inner-light and the meditator. Thus the physical reality of the inner-light in relation to the meditator perceiving the light is preserved. But it does not provide the element of physical reality of inner-light system to another observer (another system in this context). So the physical reality of inner-light is not there for the second observer. But again the meditator can share information with other observer by means of words or expression. This will be a topic of epistemological reality rather than ontological reality. The following figure is a description of the same.

\section{Inner Light Perception In D'espagnat Position}

The following statement summarises the D'Espagnat (D'Espagnat, 2006) philosophy regarding the physical realism:

"...defines his philosophical view as open realism; existence precedes knowledge; something exists independently of us even if it cannot be described".

Thus as per this theory, the physical reality exists but we are unable to describe it. 
A closer look towards this theory brings us close to the theory of critical realism which propounds that there are aspects of reality which can not be recorded by senses. But the reality exists. Going by this theory, it explains the situation of both the observers in our inner-light-perception condition. The other observer cannot access the reality of the inner-light but the inner-light exists independent of this inability of the other observer. However, as Vernette and Caponigro quote, perhaps this theory departs from the concept of physical realism itself.

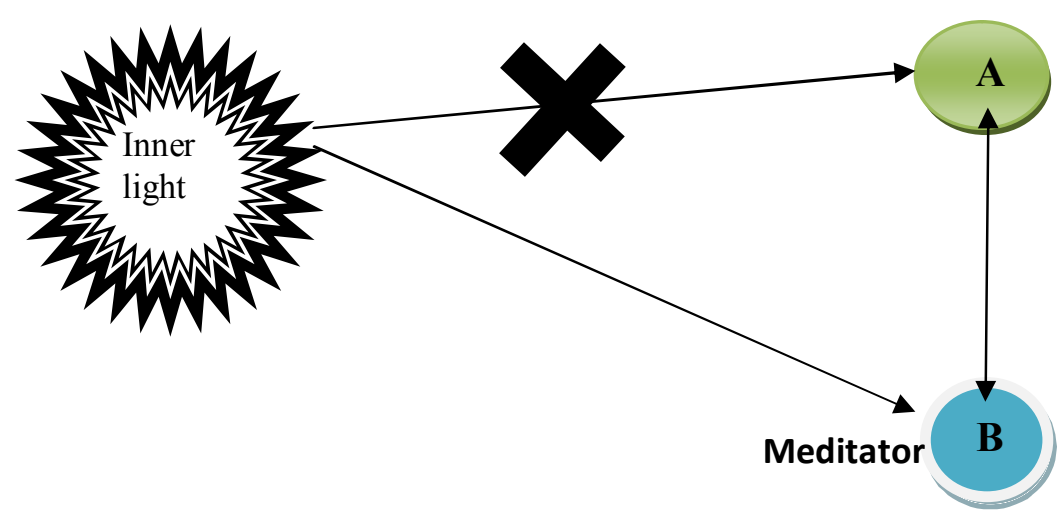

The cross represents the inability of the other observer other than the meditator to access the information regarding the system of the inner-light. Thus the physical realism for the inner-light system to observer system $B$ is possible as he is the meditator but the physical realism of inner light system is not possible for system A. However, the exchange of information regarding the inner-light system between systems $A$ and $B$ is possible.

Inner-Light Perception In Zeilinger'S Position Zeilinger introduced the individuality concept in for the purpose of description of physical realism. His theory is as follows:

"First we note that our description of the physical world is represented by propositions. Any physical object can be described by a set of true propositions. Second, we have knowledge or information about an object only through observations. It does not make any sense to talk about reality without the information about it. Any complex object which is represented by numerous propositions can be decomposed into constituent systems which need fewer propositions to be specified. The process of subdividing reaches its limit when the individual subsystems only represent a single proposition, and such a system is denoted as an elementary system. (qubit of modern quantum physics)." (Kofler, 2006)
Going by this theory, our condition of "inner-light-perception" gets the status of physical realism. As per this theory, there is no need for describing physical realism of the inner-light perception for the meditator because he is observing it directly. For the other observer, the physical realism of the inner-light is possible by a representation of a true set of propositions. Whether he reaches the stage of single proposition will depend on the method of decomposition of the propositions into constituent systems. But the physical reality of the state can be represented to him by the set of propositions.

As is evident from the above descriptions, as we move from the Einstein's concept to the Zeilinger's concept, the phenomenon of 'inner-light perception' can be proved to be physically real. We chose to focus on the inner light because it represents a unique subjective state experienced by few people and is not pathological like auditory hallucinations, so can not be directly correlated to any 
neurochemical alteration of brain. Such states can be many like dream states, imagination states etc but inner light is again different from them because it is specifically related to a procedure known as meditation, whose reality (both physical and critical) is again an arguable issue.

\section{The Coincidence of Physical and Critical Realism: Subjective Experiences}

Here, we can see an obvious similarity between the physical realism and critical realism concepts. The subjective experiences like the inner-light perception can be seen as the quantum information process which is the empirical level of reality in terms of the Roy Bhaskars critical realism philosophy. Then the apparent randomness can be seen as the reason of differences between the empirical levels of reality. Perhaps this randomness is then generated by the mechanisms of the real level causing the events of factual level of reality to occur. Thus the subjective experience of inner-light here act as the 'basic units' in Zeilienger's concept and of immanent experience in Bhaskar's concept. However, as Bhaskar pointed out, to reach the real level, one has to transcend the immanent level of experience. The exact correlate of this transcendence in physical realism can not be pointed out at present but it is very obvious that the immanent experience which creates the empirical level of reality in Bhaskar's concept corresponds to the individual reality concept of Zeilinger's philosophy taking into account the randomness created by the basic units. We suggest that the subjective experience (i.e. inner-light) can pick up elements of the underlying reality. On this basis, we argue about a new form of realism. Realism linked with the "choice" of an individual experience. The apparent randomness of data picked up in different experiences is seen as parts of the big puzzle of the underlying reality.

\section{The Ontic Status of The Quantum Information Theory}

So after notoriously vigorous efforts, we have proved that the inner-light perception is compatible with the laws of physical reality. But does that mean that it is physically real? It would not be an appropriate scientific approach to call it physically real until we give a theoretical explanation for it. For this theoretical explanation, we turn towards quantum mechanics: the quantum information theory. We will see whether what we are looking for explaining this and similar unique subjective states lies in the realms of quantum mechanics in the form of the information concept.

Recently, with the development of quantum information theory, several scientists have given to the information a fundamental role in the description of the Nature.

Quantum information theory has led to new way to look at the foundations of QM, including a greater emphasis on possible role of subjective probability in QM. Several works claims that the quantum mechanics can be viewed as an information theory. According to these works, the description of physical systems in terms of information and information processing, is the only way to describe physical system. For instance, according Bub's words (Bub, 2004): I argue that quantum mechanics is fundamentally a theory about the representation and manipulation of information, not a theory about the mechanics of nonclassical waves or particles. The notion of quantum information is to be understood as a new physical primitive. The authors give at the information an ontic statute. In this context it is possible, for instance, to deduce the physical laws and the matter from the information. Others extreme positions (Zeilinger, 2001) claim that: "The discovery that individual events are irreducibly random is probably one of the most significant findings of the twentieth century, even for single particles, it is not always possible to assign definite measurement outcomes independently of and prior to the selection of specific measurement apparatus in the specific experiment. For this reason, the distinction between reality and our knowledge of reality, between reality and information, cannot be made.

All these approaches (quantum theoretic description of physical systems) start in general from the assumption that we live in a world in which there are certain constraints on the acquisition, representation, and communication of information. According to them, the description of physical systems in terms of information and information processing, is 
complementary (or the only way) to the conventional description of physical system in terms of the laws of physics.

The notion of quantum information is to be understood as a new physical primitive. The primitive role of the information seems to explain, according to some authors, the deep nature of physical reality. In this context, the description of a state of a quantum system is a description of the information possessed by the observer about the system. The quantum state seem a construct of the observer.

So, does quantum information theory finally help us to resolve the conceptual of quantum mechanics? And does the theory indicate a new way of thinking about the world one in which the material as the fundamental subject matter of physical theory is seen to be replaced by the immaterial: information?

\section{Inner-Light Perception As A Quantum Process In Brain: Deductions of Zeilingers 'Information' Concept}

Our discussion till now has focussed on the compatibility of inner light perception with the principles of critical realism, physical realism and quantum information theory. We now proceed to explore the possibilities of considering innerlight perception as quantum process in brain. The very idea of this consideration could seem inappropriate keeping in view the differences in the magnitude and scale of the two events. However, we proceed with this exploration and will see that how the two of them can satisfactorily be correlated once we focus on Zeilinger's interpretation of quantum mechanics. We suggest that he provides a good support for our thesis. According to Zeilinger to Brukner (2001) the information is the most fundamental notion in quantum mechanics. Based on this observation they suggest new ideas for a foundational principle for quantum theory. They proposed, that the foundational principle for quantum theory may be identified through the assumption that the most elementary system carries one bit of information only.

Therefore an elementary system can only give a definite answer in one specific measurement. The irreducible randomness of individual outcomes in other measurements and quantum complementarity are then necessary consequences.

Moreover, they affirm that the objective randomness of the individual quantum event is a necessity of a description of the world in view of the significant influence the observer in quantum mechanics has". They claim that "whatever information could be ascribed to 'basic units' on the quantum level had to be subjective because it depended on the observer's choice". Now, can we consider the inner-light state as an individual quantum process in the brain? Based on these premise, we argue that our researches are closely related to Zeilinger's view. Then there are three obvious deductions of this theory:

a) The inner light (or the state before it in time) could be a state of quantum superposition in brain. This could be explained by any one of the several quantum theories of consciousness (Stapp, 2004).

b) This occurrence of inner-light would be present irrespective of the meditators observation of it. This can be seen similar to several quantum states where conscious observations are not involved. For example, progression of the state as per the Schrödinger's prediction when the collapse due to conscious observation is not involved.

c) Then the perception of inner-light would be seen as the collapse of the quantum superposition. The way this collapse (Copenhagen or Everetts multiple worlds theory or Orchestrated objective reduction by Penrose-Hameroff etc) occurs is very difficult to conceptualise at present. We will not go in to the depths of this collapse phenomena because this article is actually meant for the purpose of describing the physical reality aspects of such a subjective phenomena.

However, these quantum interpretations are just suppositions and far from making any theoretical statements at present. We just mentioned them to propose a similarity between this inner-light perception and a quantum process in brain. In fact, we will at the end of this discussion 
turn towards the problems that classical Copenhagen interpretation pose for such a measurement. We think that, our approaches are simply attempts to keep, in one way or other, a realistic view of the world. Probably, quantum physics will be superseded by a new theory, but it is likely that this will be much more radical than anything we have today. This radical view, we retain will include the "reality" of individual experience.

\section{Turning Towards: The Copenhagen Interpretation}

Any discussion on the philosophy of quantum mechanics will be incomplete until we address the Copenhagen interpretation. Thus we conclude this discussion with by presenting our proposition of inner-light perception phenomenon in the light of the classical Copenhagen interpretation of quantum mechanics. Historically, the understanding of the mathematical structure of QM went through various stages. Very briefly, the Copenhagen interpretation assumes two processes influencing the wavefunction, namely, 1) its unitary evolution according to the Schrödinger equation, and 2) the process of measurement. In other words, quantum mechanics is problematic in the sense that it is incomplete and needs the notion of a classical device measuring quantum observables as an important ingredient of the theory. Due to this, one accepts that there exist two worlds: the classical one and the quantum one. In the classical world, the measurements of classical observables are produced by classical devices. In the framework of standard theory, the measurements of quantum observables are produced by classical devices, too. Due to this, the theory of quantum measurements is considered as something very specifically different from classical measurements.

As we know, the problem of the realism of physical theories is quite complex. The contemporary of scientific community admit without hesitation that the existence of the objective physical reality is a condition sine qua non for any scientific activity. This stance seems to correlate with the structure of classical physics where each measured physical quantity finds its counterpart in the external reality. This means that one can always assign an element of the physical reality to any value obtained in an experiment. In other words, the physical system is said to possess a certain property (Vernette, 2006). Most interpretations of quantum mechanics are focused on the issue of the measurement problem, that is related of the inability to assign a single value of an observable obtained in a measurement to a given quantum system described by means of a wave function. Moreover, the matter of the emergence of the macroscopic reality out of the quantum regime needs to be addressed in this context as well. The lack of the straightforward relation between the values of the observables and the properties of a microscopic system under study is a consequence of the perturbation of the state of the system by the process of a measurement. However, this cannot be regarded as a valid criterion to deny the real existence of quantum states indicating that in quantum mechanics the states of the systems under study are not directly measurable but they are as real as those in the classical regime. The contemporary attitude towards quantum mechanics reflects much of the legacy of the Copenhagen interpretation where the quantum formalism is treated exclusively as a tool to calculate probabilities of obtaining certain values of observables and not referring to any real quantum states.

After the proposition that inner-light perception is a quantum state in brain, we now correlate the problem of measurement as applicable to this condition in the same way it is applicable to the Copenhagen interpretation of Quantum world as a whole. But as mentioned above, this inability to measure the quantum state is not a valid criteria to rule out the reality of such a possible quantum state. Here we see another coincidence of the theories of physical and critical realities where the (consciously) obtained information is not the real level of reality, instead it represents only the empirical level (Bhaskar, 1978). 


\section{References}

Bhaskar R. A Realist Theory of Science, London: Verso2008

Bhaskar R. A Realist Theory of Science. Hassocks, West Sussex: Harvester Press, 1978.

Brukner C, Zukowski M, ZeilingerA. The essence of entanglement. arXiv:quant-ph/0106119 2001

Bub J. Quantum mechanics is about quantum information, Foundations of Physics Festschrift issue for James Cushing (2004)

Collier, Critical Realism: An Introduction to Roy Bhaskar's Philosophy. London: Verso, 1994: 16-25.

Dennett DC. "Quining qualia", in Consciousness in Contemporary Science, edited by A. Marcel \& E. Bisiach, Oxford University Press. 1988.

Dennett DC. Consciousness Explained. Little, Brown, and Company, 1991.

d'Espagnat B. On physics and philosophy. Princeton University Press 2006

Einstein B, Podolsky N. Rosen Phys. Rev. 1935;47: 777.

Fade S Using interpretative phenomenological analysis for public health nutrition and dietetic research: a practical guide. Proceedings of the Nutrition Society 2004;63:647-653.

Fiske ST \& Taylor SE Social Cognition, 2nd ed. New York: McGraw-Hill 1991.

Kofler J, Zeilinger A. Sciences et Avenir Hors-Srie, October/November 2006;148

Prakash R, Haq MZU, Prakash O,Sarkhel S \&Kumar D. Inner light perception of Vihangam Yogis-A qualitative study. Journal of Consciousness studies. Article in press.

Prasad R. Introduction to Vihangam Yoga . Published by: Adhyatmic Yantralaya, Garwar, Dist- Ballia, U.P (India) 1989

Prasad R. Towards God Realization. Published by: Adhyatmic Yantralaya, Garwar, Dist- Ballia, U.P (India). 1994

Reed M. 'Reflections on the Realist Turn in Organization and Management Studies'. Journal of Management Studies2005;42:1621-1644.

Reid K, Flowers P, Larkin M. Exploring lived experience The Psychologist 2005; 18(1):20-23.

Rovelli C. Relational quantum mechanics, Intl. J. theor. Phys. 1996; 35:1637-1678

Stapp HP "Quantum Mechanical Theories of Consciousness" Lawrence Berkeley National Laboratory. Paper LBNL-56281. (August 16, 2004). http://repositories.cdlib.org/lbnl/LBNL-56281

Tsang EW, KwanK. Replication and Theory Development in Organizational Science: A Critical Realist Perspective. Academy of Management Review 1999; 24 (4):759- 780

Vernette D, Caponigro M. "Physical quantity" and " Physical reality" in Quantum Mechanics: an epistemological path. arXiv:quant-ph/0612036v1 5 2006 